\title{
Validation of a Single-Nucleotide Polymorphism-Based Non-Invasive Prenatal Test in Twin Gestations: Determination of Zygosity, Individual Fetal Sex, and Fetal Aneuploidy
}

\author{
Errol R. Norwitz ${ }^{1}$, Gabriel McNeill ${ }^{2}$, Akshita Kalyan ${ }^{2}$, Elizabeth Rivers ${ }^{2}$, Ebad Ahmed ${ }^{2}$, \\ Ling Meng ${ }^{2}$, Phikhanh $\mathrm{Vu}^{2}$, Melissa Egbert ${ }^{2}{ }^{-}$, Marlene Shapira ${ }^{2}$, Katie Kobara ${ }^{2,+}$, \\ Sheetal Parmar ${ }^{2}$, Shruti Goel ${ }^{2}$, Sarah A. Prins ${ }^{2, \pm(1)}$, Israel Aruh ${ }^{3}$, Nicola Persico ${ }^{4}$, \\ Jared C. Robins ${ }^{5}$, Brian Kirshon ${ }^{6}$, Zachary P. Demko ${ }^{2}$, Allison Ryan ${ }^{2}$, Paul R. Billings ${ }^{2}$, \\ Matthew Rabinowitz ${ }^{2}$, Peter Benn ${ }^{7}$, Kimberly A. Martin ${ }^{2}$ and Herman L. Hedriana ${ }^{2,8, *(\mathbb{D})}$ \\ 1 Tufts Medical Center and Tufts University School of Medicine, Boston, MA 02111, USA \\ Natera, Inc., San Carlos, CA 94070, USA \\ Dr. Israel Aruh's IVF and Infertility Clinic, 35220 Izmir, Turkey \\ Ospedale Maggiore Policlinico, 20122 Milan, Italy \\ Northwestern University, Feinberg School of Medicine, Chicago, IL 60611, USA \\ Houston Perinatal Associates, Houston, TX 77054, USA \\ UConn Health, Farmington, CT 06030, USA \\ University of California Davis Health, Sacramento, CA 95819, USA \\ * Correspondence: hlhedriana@ucdavis.edu \\ † Present Address: Palo Alto Medical Foundation, San Carlos, CA 94070, USA. \\ $\ddagger$ Present Address: Grail, Inc., Menlo Park, CA 94025, USA.
}

Received: 22 May 2019; Accepted: 26 June 2019; Published: 28 June 2019

\begin{abstract}
We analyzed maternal plasma cell-free DNA samples from twin pregnancies in a prospective blinded study to validate a single-nucleotide polymorphism (SNP)-based non-invasive prenatal test (NIPT) for zygosity, fetal sex, and aneuploidy. Zygosity was evaluated by looking for either one or two fetal genome complements, fetal sex was evaluated by evaluating Y-chromosome loci, and aneuploidy was assessed through SNP ratios. Zygosity was correctly predicted in $100 \%$ of cases (93/93; 95\% confidence interval (CI) $96.1 \%-100 \%$ ). Individual fetal sex for both twins was also called with $100 \%$ accuracy $(102 / 102 ; 95 \%$ weighted CI $95.2 \%-100 \%)$. All cases with copy number truth were also correctly identified. The dizygotic aneuploidy sensitivity was 100\% (10/10; $95 \%$ CI $69.2 \%-100 \%)$, and overall specificity was $100 \%(96 / 96 ; 95 \%$ weighted CI, $94.8 \%-100 \%)$. The mean fetal fraction (FF) of monozygotic twins ( $n=43$ ) was $13.0 \%$ (standard deviation (SD), $4.5 \%)$; for dizygotic twins $(n=79)$, the mean lower FF was $6.5 \%$ (SD, 3.1\%) and the mean higher FF was $8.1 \%(\mathrm{SD}, 3.5 \%)$. We conclude SNP-based NIPT for zygosity is of value when chorionicity is uncertain or anomalies are identified. Zygosity, fetal sex, and aneuploidy are complementary evaluations that can be carried out on the same specimen as early as 9 weeks' gestation.
\end{abstract}

Keywords: non-invasive prenatal testing; twins; zygosity; aneuploidy; Down syndrome; prenatal screening; chorionicity

\section{Introduction}

Twin gestations account for approximately 1 in 30 live births in the United States [1] and have a 4- to 10 -fold increased risk of perinatal complications compared to singleton pregnancies [2]. Monochorionic (MC) twins account for $\sim 20 \%$ of twin gestations [3,4] and have higher rates of major structural defects, 
miscarriage, preterm delivery, and selective fetal growth restriction compared to dichorionic (DC) twins [5]. Of particular importance, $\mathrm{MC}$ twins are at high risk for twin-to-twin transfusion syndrome (TTTS), which accounts for more than one-third of perinatal MC twin deaths [6,7].

Early establishment of zygosity can prompt increased surveillance of cases at high risk for TTTS and other MC twin-related abnormalities [8]. Ultrasound examination performed in the first trimester or early second trimester can distinguish between MC and DC twins, but this is less reliable later in pregnancy [9]. Because dizygotic (DZ) (non-identical) twins almost always have a DC placenta, establishing zygosity through genetic testing could potentially aid in patient management. An accurate non-invasive prenatal test (NIPT) for zygosity would be particularly advantageous for women presenting later in pregnancy, when there is a fetal abnormality or aneuploidy marker in one of a same-sex pair, or where there is MC/DC uncertainty based on an early ultrasound examination. Accurate establishment of zygosity can be achieved through analysis of single-nucleotide polymorphisms (SNPs) from whole blood, dried blood spots, and saliva [10] and an initial proof-of-principle report demonstrated the feasibility of prenatal cfDNA-based zygosity determination $(n=8)$ [11].

Zygosity testing and the evaluation of risk for fetal aneuploidy can be conducted on the same sample. Aneuploidy screening is of particular value in DZ pregnancies because the per pregnancy risk (i.e., the probability that at least one fetus will be affected) is increased relative to the risk in singleton pregnancies [12,13]. Moreover, DZ twinning is more common in older women and these women will therefore have higher prior risk [1].

Non-invasive prenatal testing (NIPT) has been developed as a screening test for fetal aneuploidy in twin pregnancies. Meta-analyses of NIPT in twin pregnancies have shown high sensitivity and specificity for trisomy 21 [14,15], with test performance superior to traditional screening methodologies [16].

In this study, we validate the performance of a single-nucleotide polymorphism (SNP)-based NIPT to assign zygosity, evaluate the accuracy of sex determination, and present preliminary data on fetal aneuploidy screening in twin gestations.

\section{Materials and Methods}

Pregnant women ( $\geq 18$ years of age) with sonographically confirmed twin pregnancies were enrolled at 21 locations in compliance with local laws and institutional review board-approved protocols (Supplementary Methods 1) (Western IRB Protocol number 20130376). Following informed consent, $20 \mathrm{~mL}$ of maternal blood was collected; samples were analyzed at a Clinical Laboratory Improvement Act (CLIA)-certified and College of American Pathologists (CAP)-accredited laboratory (Natera, Inc.; San Carlos, CA, USA) using an SNP-based NIPT methodology described previously [17-19]. Samples were accumulated from April 2013 to February 2017 and held frozen prior to this prospective trial. All cases and the corresponding independently determined confirmatory data for zygosity, fetal sex, and aneuploidy status of the pregnancies (Supplementary Methods 2) were de-identified prior to NIPT analysis such that the testing was carried out in a blinded manner.

Zygosity, aneuploidy status, and fetal sex NIPT calls were made using a version of a previously validated algorithm for singleton pregnancies [17-20], with modifications for twin gestations that were prespecified prior to initiation of the current validation study. All cases meeting the test eligibility criteria were included in the study. Not all cases had confirmation data for all three test components (zygosity, fetal sex, and aneuploidy); only cases with confirmatory data for a given component were evaluated for that indication. Thus, there were three overlapping cohorts consisting of 95 (30 monozygotic (MZ), 65 DZ) cases for zygosity determination, 103 (40 male/male, 35 male/female, $28 \mathrm{female} / \mathrm{female}$ ) cases for fetal sex determination, and 117 cases (11 aneuploid and 106 euploid cases) for aneuploidy testing. The total number of twin pregnancies in the study was 126 (Supplementary Table S1 and Supplementary Figure S1). Patient demographics and characteristics are detailed in Supplementary Table S2. Samples with multiple aneuploidy conditions within a twin pregnancy were excluded. 
The sensitivity, specificity, and accuracy of the test in detecting zygosity, aneuploidy, and individual fetal sex were determined on samples that received a call. First, zygosity testing was performed; subsequently, analysis for fetal sex and aneuploidy was carried out with prior risk adjusted based on zygosity status (based on clinical truth or, if it was not available, the output of the SNP-based algorithm). Overall performance metrics were estimated using a weighted average based on the population prevalence of MZ:DZ twin pregnancies (30:70) [21]. Study analysis was conducted at the laboratory using a commercial (non-research) software environment. Calls were generated by the algorithm and data were reviewed when necessary according to a predetermined standard operating procedure.

Zygosity calls were made by evaluating up to 12,568 SNPs with a pattern compatible with a single set of fetal alleles indicative of an MZ pregnancy or, alternatively, a pattern compatible with two sets of fetal alleles for a DZ pregnancy. Figure 1 illustrates how the allele distributions differ between monozygotic and dizygotic twins and explains how aneuploidy and fetal fraction can be determined from these data. For each sample, the SNPs are evaluated against the two hypotheses: first, that they are from an MZ, and second, a DZ pregnancy. For an MZ pregnancy, the interpretation of the SNP pattern is essentially the same as for a singleton pregnancy. For a DZ pregnancy, the non-maternal alleles are interpreted as being derived from one or both fetuses. A paternal sample was not required for the test interpretation. MZ sensitivity was estimated as the proportion of confirmed MZ pregnancies with a valid zygosity call that were called correctly. DZ sensitivity was estimated as the proportion of confirmed DZ pregnancies with a valid zygosity call that were called correctly. Zygosity test accuracy was estimated as the proportion of pregnancies with a valid zygosity call that were called correctly.

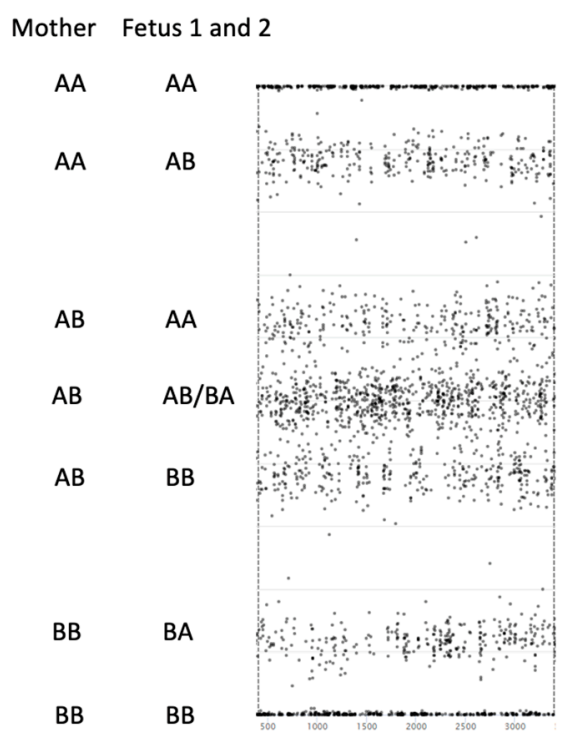

(a) Monozygotic, disomy

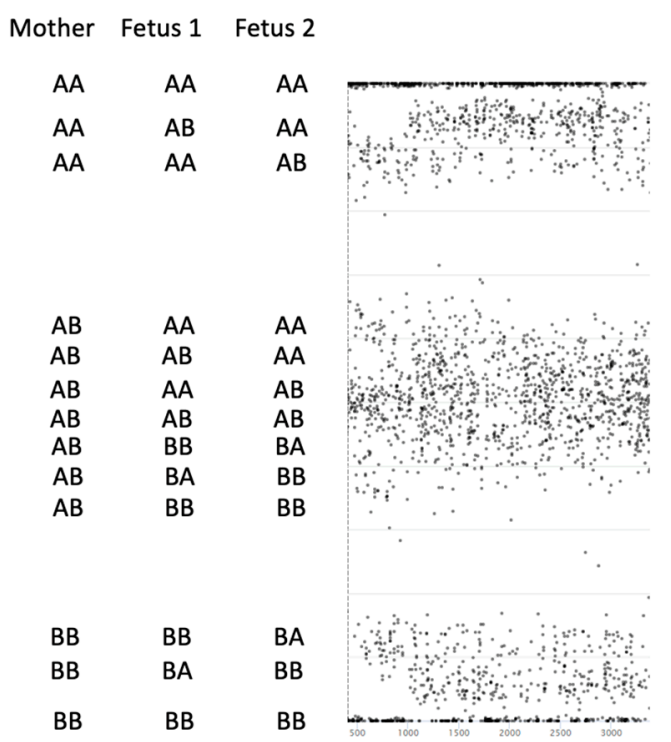

(b) Dizygotic, disomy

Figure 1. Cont. 


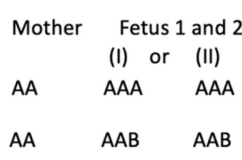

$\begin{array}{lll}A B & A A A & \\ A B & & A B A \\ A B & A A B & \\ A B & A B B & \\ A B & & A B B \\ A B & B B B & \end{array}$

$\begin{array}{lll}\text { BB } & \text { BBA } & \text { BBA } \\ \text { BB } & \text { BBB } & \text { BBB }\end{array}$

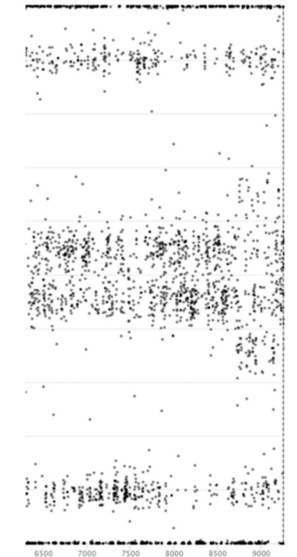

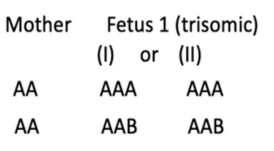

$\begin{array}{lll}A B & & \\ A B & A A B & A B A \\ A B & A B B & \\ A B & & A B B \\ A B & B B B & \end{array}$

(c) Monozygotic, trisomy
BB $\quad$ BBA $\quad$ BBA

BB $\quad$ BBB $\quad$ BBB

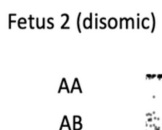

$A A$

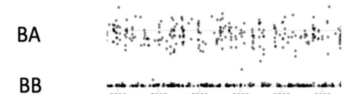

(d) Dizygotic, one trisomic fetus

Figure 1. Heterozygosity plots for SNP alleles for a single chromosome in twins. Legend: $x$-axisrelative position of the SNP along the chromosome; $y$-axis-relative amount of the alleles (100\% A at the top and 100\% B allele at the bottom). (a) The pattern of SNPs in monozygotic twins is the same as in singleton pregnancies as there are only two different genotypes present. The relative vertical position of heterozygous alleles is determined by the fetal fraction, with two SNP bands at the top, three in the middle, and two at the bottom. (b) Dizygotic twins share zero, one, or two homologous regions of their genomes, depending on the recombination and inheritance patterns. The pattern for dizygotic twins differs from monozygotic twins over those regions where the twins share zero or one homologous regions due to the extra genotype present in the DNA mixture. Over those regions, there are more possible combinations alleles at a given SNP, meaning more bands. In this example, the fetal fraction for fetus 1 is less than that for fetus 2. (c) When trisomy is present in a monozygotic twin pregnancy, the number of bands change, with the number depending on the number of homologous regions from each parent. The pattern is similar to a singleton pregnancy where trisomy is present. Two alternative fetal genotypes are shown; I depict the situation where two homologs are inherited from the father, or where two identical homologs are inherited from the mother, and II is where one of each of the maternal homologs are present in the fetus. The pattern can change along the chromosome as a result of recombination. (d) The combination of a trisomy and disomy in a dizygotic twin pregnancy results in a complex pattern. Although more complex, the informatics can compute the two FFs and determine the presence of trisomy through comparison of relative SNP amounts across chromosomes.

Fetal fraction (FF) estimates were determined for both fetuses based on the allele ratios: A combined FF for MZ twins using the same methodology as for singletons and two distinct FF estimates for DZ twins. An FF $<2.8 \%$ for a putative DZ fetus or a combined FF $<2.8 \%$ for a putative MZ pregnancy was too low for the test to provide a result.

Fetal sex assessment was based on the analysis of 277 Y-chromosome loci. The number of male and female fetuses in each twin pregnancy was determined by relating the proportion of $Y$ chromosomal DNA to the observed FF. Two males were called when the amount of $Y$ chromosomal DNA was consistent with the sum of FFs; one male and one female were called when the amount of Y chromosomal DNA was consistent with one of the FFs; two females were called when no Y chromosomal DNA was detected. MZ fetal sex accuracy was defined as the proportion of MZ samples with a valid call that were correctly called as "two males" or "two females" for which confirmed gender information on both fetuses was available. DZ fetal sex accuracy was defined as the proportion of DZ pregnancies with gender truth on both fetuses that received a valid call and were correctly called as either "two males," "one male and one female," or "two females." The overall fetal sex accuracy was computed 
using the weighted average of MZ and DZ cases that received a call. Fetal sex was only analyzed for cases with an aneuploidy result.

Aneuploidy assessment in MZ twins followed the same methodology used for singleton pregnancies [22]. DZ twins were only assessed for trisomies 13, 18, and 21. A priori risk for an aneuploid pregnancy was based on maternal age-specific rates for singleton pregnancies, with adjustments based on the assigned zygosity (Supplementary Methods 3) [12]. A risk of $>1 / 100$ for trisomy 21, trisomy 18 , or trisomy 13 was considered a high-risk result. A pregnancy was considered "screen positive" if a copy number abnormality was suspected in at least one fetus and "screen negative" when no abnormality was suspected in either fetus; the methodology did not assign a fetus-specific aneuploidy risk. Aneuploidy sensitivity and specificity (MZ and DZ) and overall aneuploidy specificity using an MZ:DZ weighted average were estimated. In addition, MZ specificity of the test in detecting monosomy $X$ was estimated using a $>1 / 100$ risk as a cut-off (Supplementary Methods 4). Generally, zygosity testing could be carried out at lower FF than is possible for aneuploidy detection. Therefore, chromosomal abnormalities were only analyzed for cases when zygosity calls were available.

Samples that did not return a result for a given test were excluded from the corresponding sensitivity, specificity, or accuracy calculations; repeat samples were not requested (Supplementary Methods 5). Confidence intervals (CI) were computed for all test performance estimates (Supplementary Methods 6).

\section{Results}

\subsection{Determination of Twin Zygosity}

To validate the performance of the test for zygosity detection in twin gestations, 95 samples with confirmed zygosity were evaluated (30 MZ and $65 \mathrm{DZ}$ ) (Figure 2). All samples that received results (MZ: 29/30 (96.7\%) and DZ: 64/65 (98.5\%)) were called correctly, yielding an observed MZ sensitivity (DZ specificity) of 100\% (29/29; 95\% CI, 88.1\%-100\%) and an MZ specificity (DZ sensitivity) of 100\% (64/64; 95\% CI, 94.4\%-100\%). The overall zygosity test correct call proportion was $100 \%$ (93/93; 95\% CI, 96.1\%-100\%). Two samples (MZ: 1/30 (3.3\%) and DZ: 1/65 (1.5\%)) did not receive results due to low $\mathrm{FF}$, generating an overall estimated no-result rate of $2.1 \%(0.3 \times 1 / 30+0.7 \times 1 / 65 ; 95 \%$ weighted CI, $0.65 \%-8.4 \%)$.

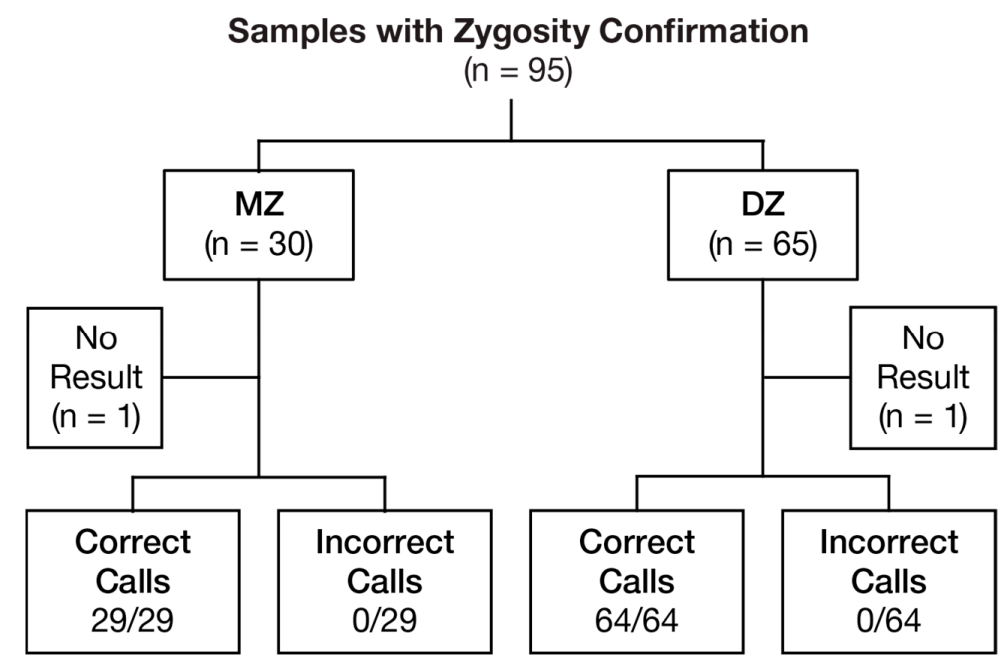

Figure 2. Zygosity determination. MZ—monozygotic; DZ—dizygotic.

\subsection{Fetal Fraction}

The mean FF of MZ twins ( $n=43)$ was $13.0 \%(\mathrm{SD}, 4.5 \%)$; for DZ twins $(n=79)$ the mean lower FF was $6.5 \%(\mathrm{SD}, 3.1 \%)$ and the mean higher $\mathrm{FF}$ was $8.1 \%(\mathrm{SD}, 3.5 \%)$. Figure 3 displays a scatterplot of the high and low FF estimates of DZ samples $(n=79)$. The Pearson correlation coefficient estimate 
between the FF estimates for each pair of FFs was 0.860 (95\% bootstrap CI, 0.736-0.937). Of the DZ cases, 20.3\% (16/79) had an FF difference of greater than $2 \%$ between the two fetuses and 11.4\% (9/79) had an FF difference of greater than $4 \%$. All cases with confirmed zygosity that were included in one or more test groups (zygosity, fetal sex, and aneuploidy) were included in the analysis.

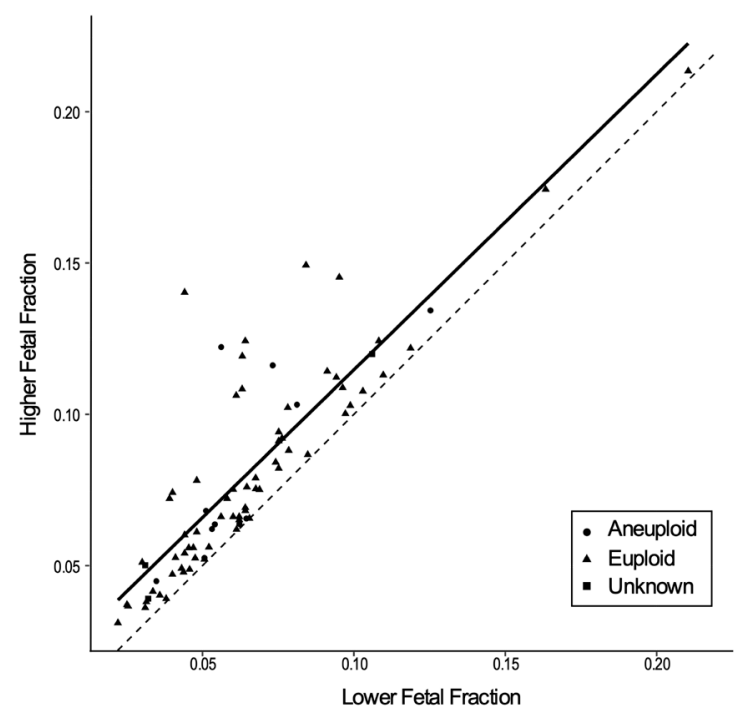

Figure 3. Scatterplot of fetal fraction estimates of dizygotic twins. Legend: Scatterplot of higher and lower fetal fraction estimates of dizygous twin samples $(n=79)$. Samples include cases that were aneuploid (circles) and euploid (triangles). Presence or absence of chromosome abnormality was unknown for three cases (squares). Aneuploid samples include cases affected with either T21, $\mathrm{T} 18$, or T13. The solid line represents the linear regression line; the dotted line represents the line of equal fetal fractions (reference 45-degree line). The Pearson Correlation Coefficient was 0.860 (95\% CI, 0.736-0.937).

\subsection{Determination of Fetal Sex}

Samples with independently determined fetal sex $(n=103)$ were evaluated to estimate the accuracy of the test for individual fetal sex determination (Figure 4). All cases that received a result were called correctly, including $40 \mathrm{MZ}$ cases (two males (20/20), two females (20/20)) and 62 DZ cases (two males (20/20), one male and one female (34/34), two females (8/8)). The overall accuracy to determine fetal sex was 100\% (102/102; 95\% weighted CI, 95.2\%-100\%). One DZ case did not receive a result due to low FF, yielding a no-result rate of $1.1 \%(0.3 \times 0 / 40+0.7 \times 1 / 63 ; 95 \%$ weighted CI, $0.03 \%-6.6 \%)$.

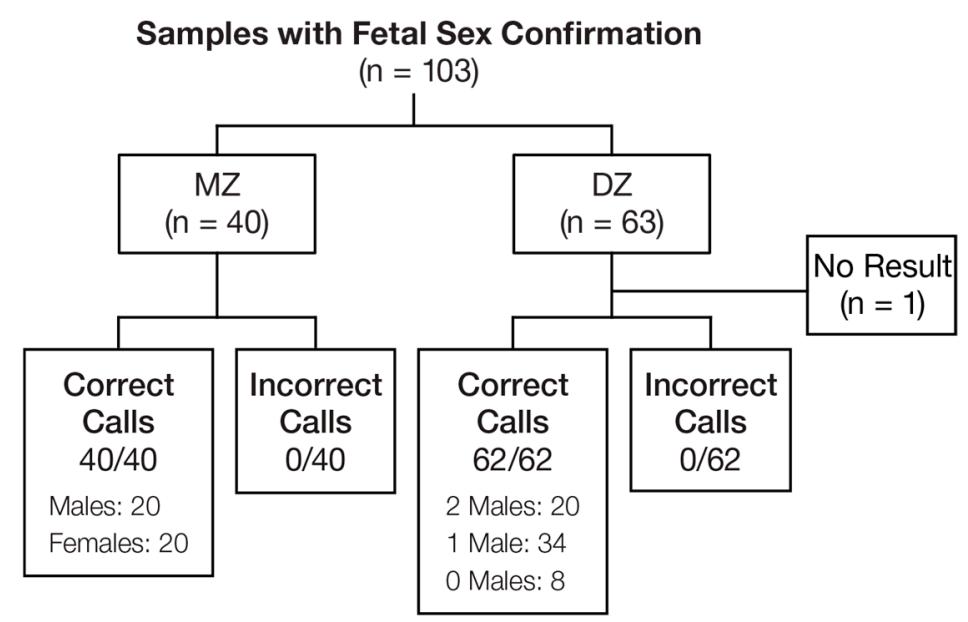

Figure 4. Fetal sex determination. MZ—monozygotic; DZ—dizygotic. 


\subsection{Screening for Chromosome Abnormality}

The evaluation of the performance of the testing for chromosomal abnormalities was based on a total of 117 cases, including 106 euploid samples, 5 T21 (1 MZ and 4 DZ), 5 T18 (all DZ), and 1 T13 (DZ) (Figure 5). All 11 aneuploidy-affected samples were correctly detected. For DZ twin gestations, the sensitivity was $100 \%$ (10/10; $95 \%$ CI, $69.2 \%-100 \%)$. Ninety-six samples (39 MZ and $57 \mathrm{DZ}$ ) that were confirmed euploid received a result; all were accurately called as negative, yielding an overall aneuploidy specificity of $100 \%$ (96/96; $95 \%$ weighted CI, $94.8 \%-100 \%)$. Of 87 euploid samples with gestational age (GA) $\geq 10$ weeks, $0 / 21(0 \%) \mathrm{MZ}$ samples and 10/66 (15.2\%) DZ samples did not receive a result, yielding an estimated overall no-result rate of $10.6 \%(0.3 \times 0 / 21+0.7 \times 10 / 66$; $95 \%$ weighted CI, $5.3 \%-19.7 \%)$.

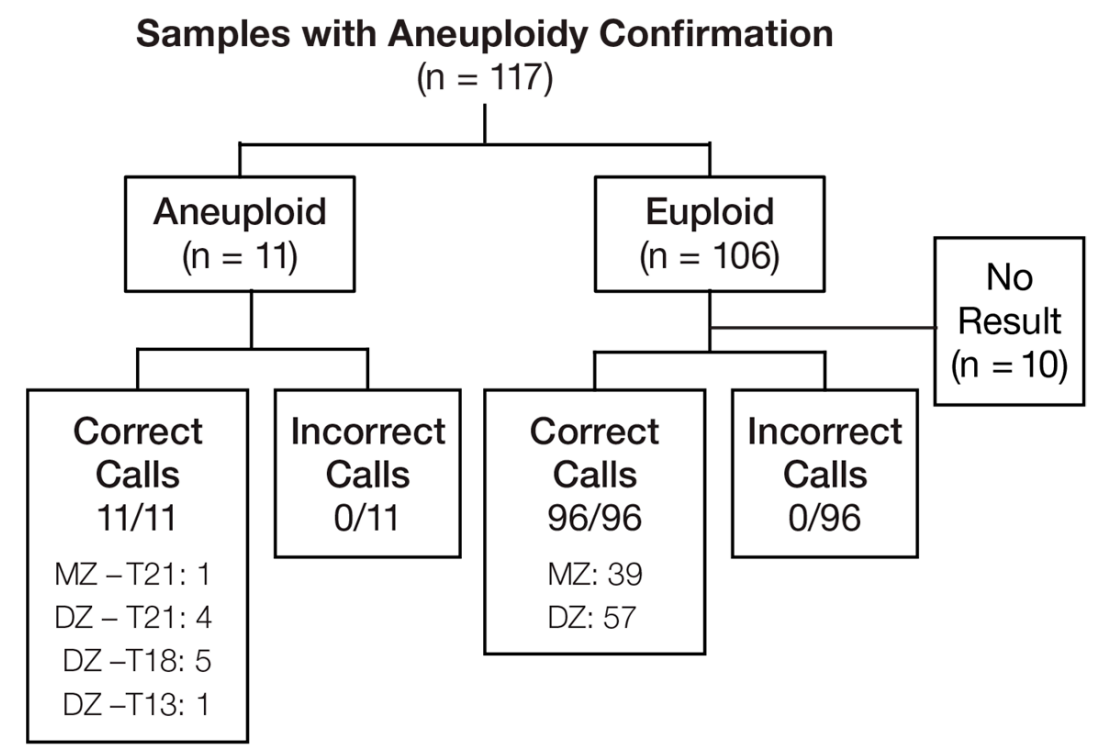

Figure 5. Aneuploidy determination. MZ—monozygotic; DZ—dizygotic.

\section{Discussion}

In this study, we report the validation of a SNP-based NIPT methodology to assign zygosity, determine individual fetal sex, and detect aneuploidy as early as 9 weeks of gestation. The three complementary pieces of information can be assessed in parallel in twin pregnancies with implications for improving prenatal management.

Accurate determination of zygosity is beneficial, particularly in women with uncertainty concerning chorionicity, which is a key factor that impacts the prognosis of twin gestations. The importance of this information early in pregnancy is well established [23]. MC twin pregnancies share their blood supply and up to $15 \%$ of these cases are affected by TTTS [7]. Furthermore, MC pregnancies are also at high risk for twin anemia-polycythemia sequence, fetal anatomic abnormalities, fetal growth restriction, and prematurity.

Monochorionic/monoamniotic (MCMA) twins, although rare $(<1 \%$ of $\mathrm{MZ}$ twins), are at the highest risk of adverse outcomes, primarily due to additional complications associated with cord entanglement [24]. MC pregnancies require complex prenatal surveillance, at a minimum of every two weeks beginning in the second trimester [25]. Twin pregnancies with dichorionic/diamniotic (DCDA) placentation have the most favorable outcome, although risk for pregnancy complications remains higher than that for singleton pregnancies.

Chorionicity assignment through ultrasound is the current gold standard during pregnancy and has high specificity and sensitivity in the first trimester. However, the accuracy of ultrasound for chorionicity establishment is both operator- and time-dependent. When performed in the late first/early second trimester, up to $19 \%$ of high-risk MC twins were incorrectly classified as DC [26]. DZ twins, 
which make up $70 \%$ of all twin pregnancies, predominantly have DC placentation. In contrast, MZ twins may either have a DC (20-30\%) or MC (70-80\%) placentation. Establishing zygosity can therefore provide an alternative source of information to assess risk in twin pregnancies and this would be of particular value for women who do not receive first trimester ultrasound. Zygosity determination needs be combined with FF determination for both fetuses in order to avoid interpreting a DZ pregnancy where one fetus has very low FF as an MZ pregnancy. We show here that the SNP-based NIPT can be used for the accurate determination of zygosity as well as determination of individual fetal sex as early as 9 weeks of gestation. To our knowledge, this is the first validated NIPT to determine zygosity.

We also report on the performance of SNP-based NIPT in screening for fetal aneuploidy in twin pregnancies. Determination of robust sensitivity estimating the detection of aneuploidies, such as T13, $\mathrm{T} 18$, and monosomy $\mathrm{X}$, is challenging due to the rarity of affected twin pregnancies. Knowledge of the zygosity status for evaluation of aneuploidy risk is advantageous because the prior risk of an affected pregnancy differs based on zygosity status [12]. In addition, the FF (combined for MZ twins and individual for DZ twins) is an important determinant of the performance of NIPT for detecting aneuploidy in twin pregnancies. For MZ twin pregnancies, we observed a mean FF of $13 \%$, which is greater than the mean FF that has previously been reported for singleton pregnancies [27]. The higher combined FF in MZ twin cases should result in the detection of aneuploidies with a performance at least as effective as that for singleton pregnancies and with a lower no-call rate. However, in DZ pregnancies, the individual FF contribution of a single fetus can be $\sim 40 \%$ lower than observed in a singleton pregnancy [27]. Moreover, each fetus in a DZ twin pregnancy can contribute different amounts of FF (up to a two-fold difference) into the maternal circulation [28]. Thus, NIPT that only measures a combined FF is more likely to result in an incorrect call for aneuploidy risk assessment in a DZ twin pregnancy. For example, if the FF of an affected fetus is below the threshold for detection, but the total FF appears satisfactory due to a high FF contribution from the euploid co-twin, reporting based on the combined FF could lead to the incorrect assignment of a low-risk result for both fetuses. This scenario is of most concern for pregnancies where one is affected by T13 or T18 because the expected FF is significantly lower than a euploid pregnancy [29]. Given that FF is an important determinant of NIPT performance in twin pregnancies, tests that do not report individual twin FF should be interpreted with caution. Conversely, as observed in this study, methods designed to determine individual FF for DZ twins will inevitably result in no-call rates that are higher compared to singleton pregnancies, since such a method will identify those cases with individual fetal fractions too low to give a confident result [30]. Additional pregnancies where results cannot be provided include donor egg pregnancies, surrogacy, and pregnancies where there is parental consanguinity. Despite these difficulties, the data presented here add to a growing volume of studies showing overall efficacy of NIPT for aneuploidy screening in twin pregnancies is comparable to that for singleton pregnancies [22] and superior to traditional screening methods for the majority of cases where there is sufficient FF for an informative result. As for singleton pregnancies, NIPT in twin pregnancies constitutes a screening test and confirmation through invasive methods is necessary.

\section{Conclusions}

Zygosity, fetal sex assignment, and aneuploidy assessment can be viewed as complementary evaluations in twin pregnancies that can be performed on the same specimen. The assignment of zygosity using SNPs improves estimation of aneuploidy risk because the prior risk is different based on zygosity status. The SNP-based approach is also expected to be advantageous over genome-wide counting methods for aneuploidy because of the assignment of individual fetus FFs. In addition, zygosity facilitates patient counseling regarding the likelihood that at least one of the fetuses in a twin gestation is affected. Furthermore, early assignment of zygosity can be aligned with the ultrasound assessment of chorionicity, which enables obstetric care providers to optimize individualized prenatal care management plans. 
Supplementary Materials: The following are available online at http://www.mdpi.com/2077-0383/8/7/937/s1: Supplementary Methods 1: Study Cohort; Supplementary Methods 2: Sources of Confirmation; Supplementary Methods 3: Prior Risk Input for Aneuploidy Assessment; Supplementary Methods 4: Aneuploidy Tests—Data Analysis; Supplementary Methods 5: No-Result Rates; Supplementary Methods 6: Confidence Intervals; Supplementary Table S1: Numbers of Cases from Each of the Participating Referral Centers; Supplementary Table S2: Patient Demographics; Supplementary Figure S1: Venn Diagram of Twin Cases Included.

Author Contributions: The statistical analysis planning and analysis of the trial were performed by G.M., E.R., A.K., and A.R. The clinical trial was designed by E.R.N., Z.P.D., P.B., K.A.M., and H.L.H., S.G., S.A.P., and P.B. were involved in the manuscript preparation. P.R.B. and M.R. were involved in funding acquisition. All other authors were involved in sample acquisition, patient contacts, local study site coordination, and gathering essential study data.

Funding: This study was funded by Natera, Inc. The funding source played a role in all aspects of the study including study design, data collection and analysis, decision to publish, and preparation of the manuscript.

Acknowledgments: The authors thank the study site coordinators, Vidya Iyer (Tufts Medical Center and Tufts University School of Medicine, Boston, MA, USA), Benedetta Ischia (Ospedale Maggiore Policlinico, Milan, Italy), Mallory Snyder (Northwestern University, Feinberg School of Medicine, Chicago, IL, USA), and Beckie Rodriguez (Houston Perinatal Associates, Houston, TX, USA) for their assistance with patient enrollment and study coordination.

Conflicts of Interest: G.M., A.K., E.R., E.A., L.M., P.V., M.E., M.S., K.K., S.P., S.G., S.A.P., Z.P.D., A.R., P.R.B., M.R., and K.A.M. are/were employees of Natera, Inc. and own stock, or options to stock, in the company. P.B. is a consultant to Natera, Inc., and owns stock options. H.L.H. was an employee of Natera, Inc., and currently does not own stock or options to stock in the company. E.R.N. and B.K. currently serve on the advisory board of Natera, Inc.; they are not employees, do not get paid, and do not own stock in the company.

\section{References}

1. Martin, J.A.; Hamilton, B.E.; Osterman, M.J.K.; Driscoll, A.K.; Drake, P. Births: Final Data for 2016. Natl. Vital Stat. Rep. 2018, 67, 1-55. [PubMed]

2. Multiple gestation pregnancy. The ESHRE Capri Workshop Group. Hum. Reprod. 2000, 15, 1856-1864.

3. Mascarenhas, M.; Kamath, M.S.; Muthukumar, K.; Mangalaraj, A.M.; Chandy, A.; Aleyamma, T. Obstetric outcomes of monochorionic pregnancies conceived following assisted reproductive technology: A retrospective study. J. Hum. Reprod. Sci. 2014, 7, 119-124. [CrossRef] [PubMed]

4. Lopriore, E.; Oepkes, D. Fetal and neonatal haematological complications in monochorionic twins. Seminars in Fetal E Neonatal Medicine 2008, 13, 231-238.

5. Simpson, L.L. Ultrasound in twins: Dichorionic and monochorionic. Semin. Perinatol. 2013, 37, $348-358$. [CrossRef]

6. $\quad$ Emery, S.P.; Bahtiyar, M.O.; Dashe, J.S.; Wilkins-Haug, L.E.; Johnson, A.; Paek, B.W.; Moon-Grady, A.J.; Skupski, D.W.; O'Brien, B.M.; Harman, C.R.; et al. The North American Fetal Therapy Network Consensus Statement: Prenatal management of uncomplicated monochorionic gestations. Obstet. Gynecol. 2015, 125, 1236-1243. [CrossRef] [PubMed]

7. Townsend, R.; Khalil, A. Ultrasound screening for complications in twin pregnancy. Semin. Fetal Neonat. Med. 2018, 23, 133-141. [CrossRef]

8. Muratore, C.S.; Carr, S.R.; Lewi, L.; Delieger, R.; Carpenter, M.; Jani, J.; Deprestb, J.A.; Luks, F.I. Survival after laser surgery for twin-to-twin transfusion syndrome: When are they out of the woods? J. Pediatr. Surg. 2009, 44, 66-69. [CrossRef]

9. Maruotti, G.M.; Saccone, G.; Morlando, M.; Martinelli, P. First-trimester ultrasound determination of chorionicity in twin gestations using the lambda sign: A systematic review and meta-analysis. Eur. J. Obstet. Gynecol. Reprod. Biol. 2016, 202, 66-70. [CrossRef]

10. Hannelius, U.G.L.; Mäkelä, V.V.; Lindstedt, A.; Zucchelli, M.; Lagerberg, C.; Tybring, G.; Kere, J.; Lindgren, C.M. Large-scale zygosity testing using single nucleotide polymorphisms. Twin Res. Hum. Genet. 2007, 10, 604-625. [CrossRef]

11. Qu, J.Z.; Leung, T.Y.; Jiang, P.; Liao, G.J.; Cheng, Y.K.; Sun, H.; Chiu, R.W.K.; Chan, K.C.A.; Lo, Y.M.D. Noninvasive prenatal determination of twin zygosity by maternal plasma DNA analysis. Clin. Chem. 2013, 59, 427-435. [CrossRef] [PubMed]

12. Sparks, T.N.; Norton, M.E.; Flessel, M.; Goldman, S.; Currier, R.J. Observed Rate of Down Syndrome in Twin Pregnancies. Obstet. Gynecol. 2016, 128, 1127-1133. [CrossRef] [PubMed] 
13. Rodis, J.F.; Egan, J.F.; Craffey, A.; Ciarleglio, L.; Greenstein, R.M.; Scorza, W.E. Calculated risk of chromosomal abnormalities in twin gestations. Obstet. Gynecol. 1990, 76, 1037-1041. [PubMed]

14. Gil, M.M.; Accurti, V.; Santacruz, B.; Plana, M.N.; Nicolaides, K.H. Analysis of cell-free DNA in maternal blood in screening for aneuploidies: Updated meta-analysis. Ultrasound Obstet. Gynecol. 2017, 50, 302-314. [CrossRef] [PubMed]

15. Liao, H.; Liu, S.; Wang, H. Performance of non-invasive prenatal screening for fetal aneuploidy in twin pregnancies: A meta-analysis. Prenat. Diagn. 2017, 37, 874-882. [CrossRef] [PubMed]

16. Madsen, H.N.; Ball, S.; Wright, D.; Torring, N.; Petersen, O.B.; Nicolaides, K.H.; Spencer, K. A reassessment of biochemical marker distributions in trisomy 21-affected and unaffected twin pregnancies in the first trimester. Ultrasound Obstet. Gynecol. 2011, 37, 38-47. [CrossRef]

17. Ravi, H.; McNeill, G.; Goel, S.; Meltzer, S.D.; Hunkapiller, N.; Ryan, A.; Levy, B.; Demko, Z.P. Validation of a SNP-based non-invasive prenatal test to detect the fetal 22q11.2 deletion in maternal plasma samples. PLoS ONE 2018, 13, e0193476. [CrossRef]

18. Ryan, A.; Hunkapiller, N.; Banjevic, M.; Vankayalapati, N.; Fong, N.; Jinnett, K.N.; Demko, Z.; Zimmermann, B.; Sigurjonsson, S.; Gross, S.J.; et al. Validation of an Enhanced Version of a Single-Nucleotide Polymorphism-Based Noninvasive Prenatal Test for Detection of Fetal Aneuploidies. Fetal Diagn. Ther. 2016, 40, 219-223. [CrossRef]

19. Zimmermann, B.; Hill, M.; Gemelos, G.; Demko, Z.; Banjevic, M.; Baner, J.; Ryan, A.; Sigurjonsson, S.; Chopra, N.; Dodd, M.; et al. Noninvasive prenatal aneuploidy testing of chromosomes 13, 18, 21, X, and Y, using targeted sequencing of polymorphic loci. Prenat. Diagn. 2012, 32, 1233-1241. [CrossRef]

20. Wapner, R.J.; Babiarz, J.E.; Levy, B.; Stosic, M.; Zimmermann, B.; Sigurjonsson, S.; Wayham, N.; Ryan, A.; Banjevic, M.; Lacroute, P.; et al. Expanding the scope of noninvasive prenatal testing: Detection of fetal microdeletion syndromes. Am. J. Obstet. Gynecol. 2015, 212, 332.e1-332.e9. [CrossRef]

21. Chasen, S.T. Twin Pregnancy: Prenatal Issues; Barss, V.A., Ed.; UpToDate: Waltham, MA, USA, 2017.

22. Pergament, E.; Cuckle, H.; Zimmermann, B.; Banjevic, M.; Sigurjonsson, S.; Ryan, A.; Hall, M.P.; Dodd, M.; Lacroute, P.; Stosic, M.; et al. Single-nucleotide polymorphism-based noninvasive prenatal screening in a high-risk and low-risk cohort. Obstet. Gynecol. 2014, 124 Pt 1, 210-218. [CrossRef]

23. Lu, J.; Cheng, Y.K.Y.; Ting, Y.H.; Law, K.M.; Leung, T.Y. Pitfalls in assessing chorioamnionicity: Novel observations and literature review. Am. J. Obstet. Gynecol. 2018, 219, 242-254. [CrossRef] [PubMed]

24. Dias, T.; Mahsud-Dornan, S.; Bhide, A.; Papageorghiou, A.T.; Thilaganathan, B. Cord entanglement and perinatal outcome in monoamniotic twin pregnancies. Ultrasound Obstet. 2010, 35, 201-204. [CrossRef] [PubMed]

25. American College of Obstetricians and Gynecologists, and Society for Maternal-Fetal Medicine. ACOG Practice Bulletin No. 144: Multifetal gestations: Twin, triplet, and higher-order multifetal pregnancies. Obstet. Gynecol. 2014, 123, 1118-1132. [CrossRef] [PubMed]

26. Blumenfeld, Y.J.; Momirova, V.; Rouse, D.J.; Caritis, S.N.; Sciscione, A.; Peaceman, A.M.; Reddy, U.M.; Varner, M.W.; Malone, F.D.; Iams, J.D.; et al. Accuracy of sonographic chorionicity classification in twin gestations. J. Ultrasound Med. 2014, 33, 2187-2192. [CrossRef] [PubMed]

27. Dar, P.; Curnow, K.J.; Gross, S.J.; Hall, M.P.; Stosic, M.; Demko, Z.; Zimmermann, B.; Hill, M.; Sigurjonsson, S.; Ryan, A.; et al. Clinical experience and follow-up with large scale single-nucleotide polymorphism-based noninvasive prenatal aneuploidy testing. Am. J. Obstet. Gynecol. 2014, 211, 527.e1-527.e17. [CrossRef] [PubMed]

28. Leung, T.Y.; Qu, J.Z.; Liao, G.J.; Jiang, P.; Cheng, Y.K.; Chan, K.C.; Chiu, R.W.K.; Lo, Y.M.D. Noninvasive twin zygosity assessment and aneuploidy detection by maternal plasma DNA sequencing. Prenat. Diagn. 2013, 33, 675-681. [CrossRef] [PubMed]

29. McKanna, T.; Ryan, A.; Krinshpun, S.; Kareht, S.; Marchand, K.; Grabarits, C.; Ali, M.; McElheny, A.; Gardiner, K.; LeChien, K.; et al. Fetal fraction-based risk algorithm for non-invasive prenatal testing: Screening for trisomy 13,18, and triploidy in women with low cell-free fetal DNA. Ultrasound Obstet. Gynecol. 2019, 53, 73-79. [CrossRef] [PubMed]

30. Sarno, L.; Revello, R.; Hanson, E.; Akolekar, R.; Nicolaides, K.H. Prospective first-trimester screening for trisomies by cell-free DNA testing of maternal blood in twin pregnancy. Ultrasound Obstet. Gynecol. 2016, 47, 705-711. [CrossRef]

(C) 2019 by the authors. Licensee MDPI, Basel, Switzerland. This article is an open access article distributed under the terms and conditions of the Creative Commons Attribution (CC BY) license (http://creativecommons.org/licenses/by/4.0/). 